\title{
Picture-Hanging Puzzles
}

\author{
Erik D. Demaine ${ }^{1}$, Martin L. Demaine ${ }^{1}$, Yair N. Minsky ${ }^{2}$, Joseph S. B. \\ Mitchell $^{3}$, Ronald L. Rivest ${ }^{1}$, and Mihai Pătraşcu ${ }^{4}$ \\ 1 MIT Computer Science and Artificial Intelligence Laboratory, 32 Vassar St., \\ Cambridge, MA 02139, USA, \{edemaine,mdemaine,rivest\}@mit.edu \\ 2 Department of Mathematics, Yale University, 10 Hillhouse Ave., \\ New Haven, CT 06520, USA, yair.minsky@yale.edu \\ 3 Department of Applied Mathematics and Statistics, State University of New York, \\ Stony Brook, NY 11794-3600, USA, jsbm@ams.sunysb.edu \\ 4 AT\&T Labs-Research, 180 Park Ave., \\ Florham Park, NJ 07932, mip@alum.mit.edu
}

\begin{abstract}
We show how to hang a picture by wrapping rope around $n$ nails, making a polynomial number of twists, such that the picture falls whenever any $k$ out of the $n$ nails get removed, and the picture remains hanging when fewer than $k$ nails get removed. This construction makes for some fun mathematical magic performances. More generally, we characterize the possible Boolean functions characterizing when the picture falls in terms of which nails get removed as all monotone Boolean functions. This construction requires an exponential number of twists in the worst case, but exponential complexity is almost always necessary for general functions.
\end{abstract}

\section{Introduction}

If you hang a picture with string looped around two nails, and then remove one of the nails, the picture still hangs around the other nail. Right? This conclusion is correct if you hang the picture around the two nails in

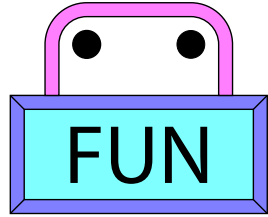

(a) A normal hanging.

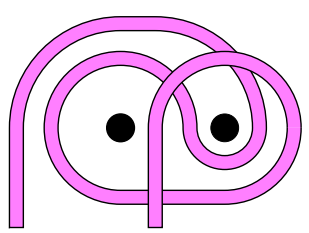

(b) Solution to the two-nail puzzle.

Fig. 1. Hanging a picture on two nails.

the obvious way shown in Figure 1(a) An intriguing puzzle, originally posed by A. Spivak in 1997 9], asks for a different hanging of the picture such that removing either nail causes the picture to fall. Figure 1(b) shows a solution.

This puzzle has since circulated around the puzzle community. Michael Hardy from Harvard posed the puzzle to Marilyn vos Savant (famous for her claimed ability to answer any riddle), and the puzzle and solution appeared in her column [12. Torsten Sillke [7] distributed the puzzle, in particular to Ed Pegg Jr., and mentioned a connection to Borromean rings and Brunnian links described in Section 3.1. This connection provides a solution to a more general form of the puzzle, which we call 1-out-of- $n$ : hang a picture on $n$ nails so that removing any 
one nail fells the picture. Pegg's MathPuzzle.com [5] has facilitated a discussion between Sillke, Neil Fitzgerald, and Chris Lusby Taylor. Fitzgerald pointed out a connection to group theory, described in Section 3.2. which provides a direct solution to the 1-out-of- $n$ puzzle. Taylor pointed out a more efficient solution to the same puzzle. All of this work is detailed and carefully analyzed in Section 3 .

We consider a more general form of the puzzle where we want the removal of certain subsets of nails to fell the picture. We show that any such puzzle has a solution: for any collection of subsets of nails, we can construct a picture hanging that falls when any entire subset of nails gets removed, but remains hanging when every subset still has at least one unremoved nail. This result generalizes picture-hanging puzzles to the maximum extent possible.

Unfortunately, our construction makes an exponential number of twists around the $n$ nails. Indeed, we show that this is necessary, for most general settings of the problem. Fortunately, we find polynomial constructions for the 1-out-of- $n$ puzzle, as well as the $k$-out-of- $n$ generalization where the picture falls only after removing (any) $k$ out of the $n$ nails. More generally, we show that any monotone Boolean function in the complexity class $\mathrm{mNC}^{1}$ (monotone logarithmic-depth bounded-fanin circuits) has a polynomial-length solution, which can also be found by a polynomial-time algorithm.

These generalizations make for fun puzzles as well as magic performances. Section 2 gives several puzzles accessible to the public that become increasingly easier to solve while reading through this paper. These constructions have been featured as a kind of mathematical magic trick during several of the first authors' talks (first his FUN 2004 plenary talk): the magician wraps large rope around various volunteers' outstretched arms (which act as the "nails"), spectators choose which arms to remove from the construction, and the magician simply "applies infinite gravity" (untangles and pulls on the ends of the rope) to cause the rope to mathemagically fall to the ground. See Figure 2 .
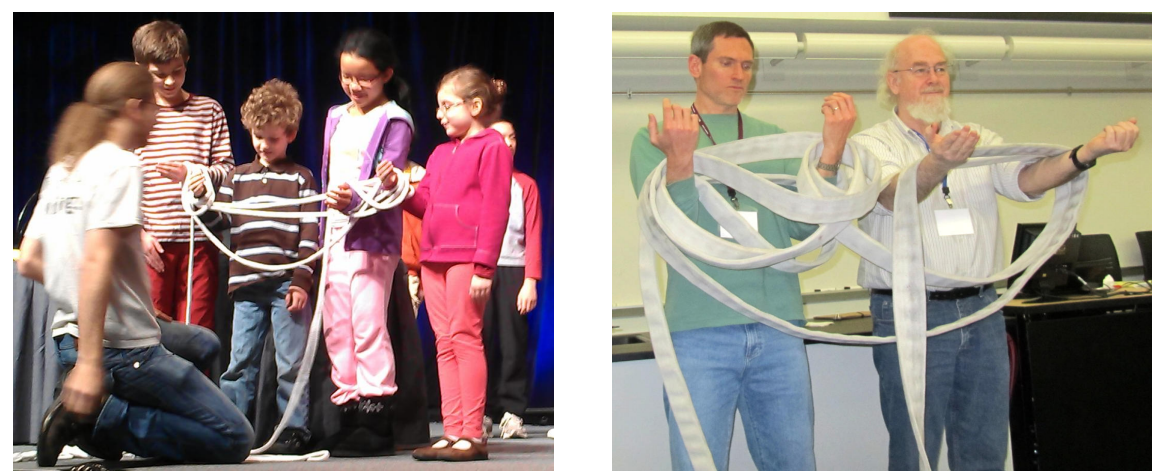

(a) A solution to Puzzle1 1 implemented by (b) A solution to Puzzle 8 implemented wrapping rope around children's arms for by wrapping fire hose from the local fire the Porter Public Lecture during the Joint department, when the first author forgot Mathematics Meetings, January 2012. To bring his rope for a Polyá Lecture, 2011.

Fig. 2. Picture-hanging puzzles performed as mathematical magic tricks. 
Our work interrelates puzzles, magic, topology, Borromean rings, Brunnian links, group theory, free groups, monotone Boolean function theory, circuit complexity, AKS sorting networks, combinatorics, and algorithms.

A related result constructs interlocked 2D polygons that separate (fall apart) when certain subsets of polygons are removed, again according to an arbitrary monotone Boolean function 2. That result is essentially a geometric analog of the topological results presented here, although most of the challenges and remaining open questions differ substantially.

\section{Puzzles}

To whet the appetite of puzzle aficionados, we present a sequence of picturehanging puzzles ranging from simple to more interesting extensions, some of which require rather involved constructions. We have tested our solutions with 38-inch lanyard wrapped around fingers, and found that this length suffices for Puzzles 1, 2, 3, 6, 7, and 8, but for the other puzzles you would need a longer cord or string. In public performances with large rope wrapped around volunteers' arms, the first author typically performs Puzzles 1, 4, 2, 6, and 8,

Puzzle 1 (1-out-of-3) Hang a picture on three nails so that removing any one nail fells the picture.

Puzzle 2 (2-out-of-3) Hang a picture on three nails so that removing any two nails fells the picture, but removing any one nail leaves the picture hanging.

Puzzle 3 (1+2-out-of-3) Hang a picture on three nails arranged along a horizontal line so that removing the leftmost nail fells the picture, as does removing the rightmost two nails, but removing one of the two rightmost nails leaves the picture hanging.

Puzzle 4 (1-out-of-4) Hang a picture on four nails so that removing any one nail fells the picture.

Puzzle 5 (2-out-of-4) Hang a picture on four nails so that removing any two nails fells the picture, but removing any one nail leaves the picture hanging.

Puzzle 6 (3-out-of-4) Hang a picture on four nails so that removing any three nails fells the picture, but removing just one or two nails leaves the picture hanging.

Puzzle 7 (2+2-out-of-2+2) Hang a picture on two red nails and two blue nails so that removing both red nails fells the picture, as does removing both blue nails, but removing one nail of each color leaves the picture hanging.

Puzzle $8(\mathbf{1 + 2}$-out-of-2+2) Hang a picture on two red nails and two blue nails so that removing any one red nail fells the picture, as does removing both blue nails, but removing just one blue nail leaves the picture hanging.

Puzzle $9(\mathbf{1}+\mathbf{3}$-out-of-3+3) Hang a picture on three red nails and three blue nails so that removing any one red nail fells the picture, as does removing all three blue nails, but removing just one or two blue nails leaves the picture hanging.

Puzzle $10(\mathbf{1 + 2 - o u t - o f - 3 + 3 )}$ Hang a picture on three red nails and three blue nails so that removing any one red nail fells the picture, as does removing any two of the blue nails, but removing just one blue nail leaves the picture hanging.

Puzzle 11 (1+1-out-of-2+2+2) Hang a picture on two red nails, two green nails, and two blue nails so that removing two nails of different colors (one red and one green, or one red and one blue, or one green and one blue) fells the picture, but removing two nails of the same color leaves the picture hanging. 


\section{Basic Theory: 1-out-of- $n$}

We start our mathematical and algorithmic study of picture-hanging puzzles with the simplest generalization, called 1-out-of- $n$, where the goal is to hang a picture on $n$ nails such that removing any one nail fells the picture. This generalization is what has been studied in the past. Our contribution is to give a thorough complexity analysis of the resulting solutions, the best of which Theorem 1 summarizes below. Then, in Section 3.4, we give a slight generalization to handle colored nails, which is enough to solve many of the puzzles listed above.

\subsection{Connection to Borromean and Brunnian Links}

According to Torsten Sillke [7, Werner Schwärzler observed that the Borromean rings provide a solution to the two-nail picture-hanging problem, and that generalized forms of Borromean rings provide solutions to more general picturehanging problems. This section describes those connections.

The classic Borromean rings are three loops that are inseparable - in topology terms, nontrivially linked - but such that no two of the rings are themselves linked. The Italian Renaissance family Borromeo's family crest draws them as interwoven circles, as in Figure 3.

The property of Borromean rings sounds similar to the picture-hanging puzzle: the three loops are linked, but removing any one loop unlinks them. Indeed, by stretching one loop to bring a point to infinity, and straightening out the loop, we can view a loop as an infinite line - or nail-that pene-

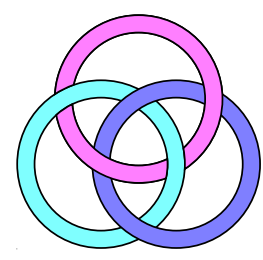

Fig. 3.

Borromean rings.

trates the entire construction. Applying this topology-preserving transformation to two out of the three loops, we convert any Borromean-ring construction into a solution to the two-nail picture-hanging puzzle. Conversely, any solution to the two-nail picture-hanging puzzle can be converted into a Borromean-ring construction by viewing the nails as infinite lines piercing the loop of rope and converting these lines to large loops.

Knot theorists have studied two generalizations to the Borromean rings. The first generalization, a Borromean link, is a collection of $n$ loops that are linked but such that no two of the loops are linked. This property seems less useful for an $n$-nail picture-hanging puzzle, because it guarantees only that removing $n-2$ of the nails fells the picture; removing between 1 and $n-3$ of the nails might fell the picture or might not, depending on the particular Borromean link at hand. The second generalization, a Brunnian link, is a collection of $n$ loops that are linked but such that the removal of any loop unlinks the rest. This property is exactly what we need for the $n$-nail picture-hanging puzzle where removing any one of the $n$ nails fells the picture. Figure 4 shows an example of transforming a Brunnian link into a picture-hanging puzzle.

Hermann Brunn [1 introduced Brunnian links in 1892, about 25 years after the first mathematical study of Borromean links [11. Brunn gave a construction for a Brunnian link of $n$ loops for every $n \geq 3$. See [6] for a more accessible 


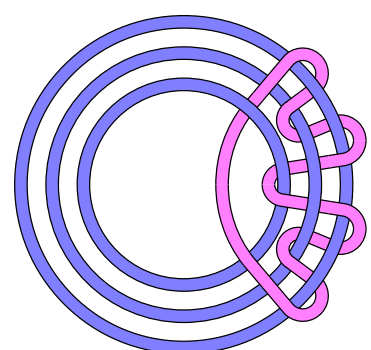

(a) Brunnian 4-link.

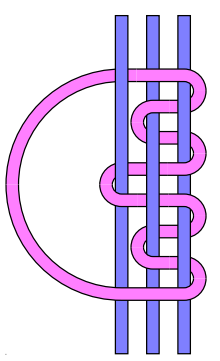

(b) Stretch-

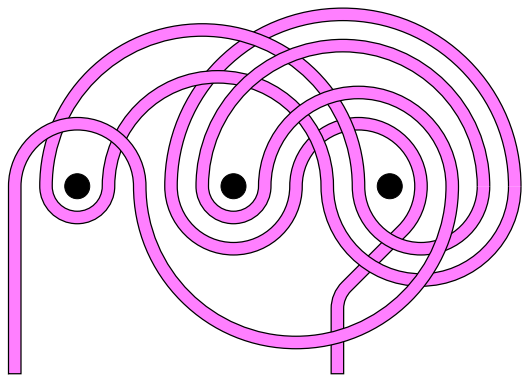

(c) Picture-hanging equivalent.

Fig. 4. Transforming a Brunnian 4-link into a 1-out-of-4 picture-hanging puzzle.

description of this construction. Using the reduction described above, we obtain a solution to the 1-out-of- $n$ picture-hanging puzzle for any $n \geq 2$. The only negative aspect of this solution is that its "size" (combinatorial complexity) grows exponentially with $n$; we will see a better solution in Section 3.3 .

Theodore Stanford [10] characterizes a generalized form of Brunnian links, where the removal of arbitrary subsets of loops causes the link to trivialize (fall apart). This problem is subtly different from picture hanging (and indeed, for years, we thought that it had already solved our problem): like Borromean links, it does not require the link remain nontrivial until one of the subsets gets entirely removed. In particular, the trivial link is considered a "solution", no matter what subsets get specified. Conceivably, Stanford's characterization can be used to obtain a solution with this property, but it is not obvious how.

\subsection{Connection to Free Group}

This section describes a more general framework to study picture-hanging puzzles in general. The framework is based on group theory and comes naturally from algebraic topology. To the best of our knowledge, this connection was first observed by Neil Fitzgerald [5]. Although we do not justify here why the grouptheoretic representation is accurate, this is an easy exercise for those familiar with algebraic topology.

A powerful way to abstract a weaving of the rope around $n$ nails uses what is called the free group on $n$ generators. Specifically, we define $2 n$ symbols: $x_{1}, x_{1}^{-1}, x_{2}, x_{2}^{-1}, \ldots, x_{n}, x_{n}^{-1}$. Each $x_{i}$ symbol represents wrapping the rope around the $i$ th nail clockwise, and each $x_{i}^{-1}$ symbol represents wrapping the rope around the $i$ th nail counterclockwise. Now a weaving of the rope can be represented by a sequence of these symbols. For example, the solution to the two-nail

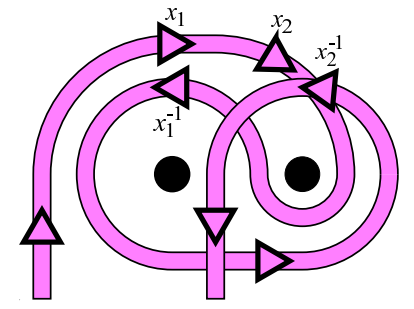

Fig. 5. Algebraic notation for Figure 1(b). 
picture-hanging puzzle shown in Figure 5 can be written $x_{1} x_{2} x_{1}^{-1} x_{2}^{-1}$ because, starting from the left, it first turns clockwise around the first (left) nail, then turns clockwise around the second (right) nail, then turns counterclockwise around the first nail, and finally turns counterclockwise around the second nail.

In this representation, removing the $i$ th nail corresponds to dropping all occurrences of $x_{i}$ and $x_{i}^{-1}$ in the sequence. Now we can see why Figure 5 disentangles when we remove either nail. For example, removing the first nail leaves just $x_{2} x_{2}^{-1}$, i.e., turning clockwise around the second nail and then immediately undoing that turn by turning counterclockwise around the same nail. In general $x_{i}$ and $x_{i}^{-1}$ cancel, so all occurrences of $x_{i} x_{i}^{-1}$ and $x_{i}^{-1} x_{i}$ can be dropped. (The free group specifies that these cancellations are all the simplifications that can be made.) Thus, the original weaving $x_{1} x_{2} x_{1}^{-1} x_{2}^{-1}$ is nontrivially linked with the nails because nothing simplifies; but if we remove either nail, everything cancels and we are left with the empty sequence, which represents the trivial weaving that is not linked with the nails (i.e., the picture falls).

In group theory, the expression $x_{1} x_{2} x_{1}^{-1} x_{2}^{-1}$ is called the commutator of $x_{1}$ and $x_{2}$, and is written $\left[x_{1}, x_{2}\right]$. The commutator is a useful tool for solving more general picture-hanging puzzles.

Terminology. In general, define a picture hanging on $n$ nails to be a word (sequence of symbols) in the free group on $n$ generators. We refer to the number of symbols in the word as the length of the hanging, as it approximates the needed length of the string or cord. The special identity word 1 represents the fallen state. Removing the $i$ th nail corresponds to removing all occurrences of $x_{i}$ and $x_{i}^{-1}$, which may or may not cause the hanging to fall.

\subsection{1-out-of- $n$}

Theorem 1. For any $n \geq 1$, there is a picture hanging on $n$ nails of length at most $2 n^{2}$ that falls upon the removal of any one nail. For each $i=1,2, \ldots, n$, symbols $x_{i}$ and $x_{i}^{-1}$ appear at most 2 n times.

Exponential construction. We start with a simpler, less-efficient construction given by Neil Fitzgerald [5] 5 The idea is to generalize the weaving $x_{1} x_{2} x_{1}^{-1} x_{2}^{-1}$ by replacing each $x_{i}$ with an inductive solution to a smaller version of the problem. In other words, we start with the solution for $n=2: S_{2}=$ $\left[x_{1}, x_{2}\right]=x_{1} x_{2} x_{1}^{-1} x_{2}^{-1}$. Now from this solution $S_{2}$ we build a solution for $n=3$ by using the same pattern but involving copies of $S_{2}$ in place of one of the $x_{i}$ 's: $S_{3}=\left[S_{2}\right.$,

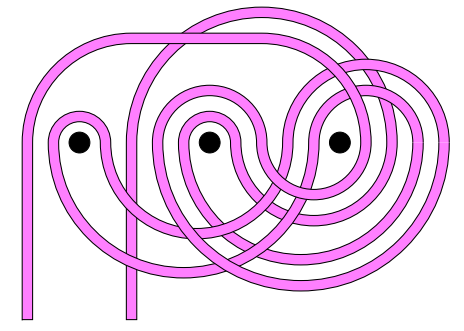

Fig. 6. Hanging a picture on three nails so that removing any one nail fells the picture.

\footnotetext{
${ }^{5}$ This construction also turns out to be essentially the same as the solution that comes out of the Brunnian-link construction described in Section 3.1 .
} 
$\left.x_{3}\right]=S_{2} x_{3} S_{2}^{-1} x_{3}^{-1}=\left(x_{1} x_{2} x_{1}^{-1} x_{2}^{-1}\right) x_{3}\left(x_{1} x_{2} x_{1}^{-1} x_{2}^{-1}\right)^{-1} x_{3}^{-1}=x_{1} x_{2} x_{1}^{-1} x_{2}^{-1} x_{3}$ $x_{2} x_{1} x_{2}^{-1} x_{1}^{-1} x_{3}^{-1}$. Here we are using the algebraic rules $(x y)^{-1}=y^{-1} x^{-1}$ and $\left(x^{-1}\right)^{-1}=x$. Figure 6 shows the corresponding picture-hanging solution.

Naturally, this construction generalizes to all $n$ by defining $S_{n}=\left[S_{n-1}, x_{n}\right]=$ $S_{n-1} x_{n} S_{n-1}^{-1} x_{n}^{-1}$. For example, $S_{4}=\left[S_{3}, x_{4}\right]=S_{3} x_{4} S_{3}^{-1} x_{4}^{-1}=x_{1} x_{2} x_{1}^{-1} x_{2}^{-1} x_{3} x_{2}$ $x_{1} x_{2}^{-1} x_{1}^{-1} x_{3}^{-1} x_{4} x_{3} x_{1} x_{2} x_{1}^{-1} x_{2}^{-1} x_{3}^{-1} x_{2} x_{1} x_{2}^{-1} x_{1}^{-1} x_{4}^{-1}$. If we remove any of the first three nails, the copies of $S_{3}$ disappear, leaving us with $x_{4} x_{4}^{-1}$ which cancels. And if we remove the fourth nail $x_{4}$, we are left with $S_{3} S_{3}^{-1}$ which cancels.

The problem with this construction, which we start to see with the full expansion of $S_{4}$, is that the length of the sequence $S_{n}$ grows exponentially with $n$. More precisely, the number of symbols in $S_{n}$ is $2^{n}+2^{n-1}-2$. To see why this count is correct, first check that $S_{2}$ has length $4=2^{2}+2^{1}-2$. Then, if we suppose inductively that $S_{n-1}$ has length $2^{n-1}+2^{n-2}-2$, we can conclude that $S_{n}$ has twice that length plus 2 for the occurrences of $x_{n}$ and $x_{n}^{-1}$, for a total of $2\left(2^{n-1}+2^{n-2}-2\right)+2=2^{n}+2^{n-1}-4+2=2^{n}+2^{n-1}-2$, as claimed.

Polynomial construction. Fortunately, there is a more efficient construction that solves the 1-out-of- $n$ picture-hanging puzzle. This construction was designed by Chris Lusby Taylor [5]. The idea is to recursively build $S_{n}$ in a more balanced way, in terms of $S_{n / 2}$ for the first half of the nails and $S_{n / 2}$ for the second half of the nails, instead of one $S_{n-1}$ and a single variable. To enable this construction, we need to consider a more general problem involving the nails from $i$ through $j$ for various $i$ and $j$. At the simplest level we have a single nail: $E(i: i)=x_{i}$. At the next simplest level we have two nails as before: $E(i: i+1)=\left[x_{i}, x_{i+1}\right]=$ $x_{i} x_{i+1} x_{i}^{-1} x_{i+1}^{-1}$. Then for an arbitrary interval $i: j$, we build $E(i: j)$ out of a recursive copy of $E$ applied to the first half of the interval and a recursive copy of $E$ applied to the second half of the interval:

$$
E(i: j)=\left[E\left(i:\left\lfloor\frac{i+j}{2}\right\rfloor\right), E\left(\left\lfloor\frac{i+j}{2}\right\rfloor+1: j\right)\right] .
$$

For $n=3$, this construction does not save anything, because splitting an interval of length three in half leaves one piece of length two and one piece of length one. But for $n=4$ we gain some efficiency:

$$
\begin{aligned}
E(1: 4) & =[E(1: 2), E(3: 4)] \\
& =E(1: 2) E(3: 4) E(1: 2)^{-1} E(3: 4)^{-1} \\
& =\left(x_{1} x_{2} x_{1}^{-1} x_{2}^{-1}\right)\left(x_{3} x_{4} x_{3}^{-1} x_{4}^{-1}\right)\left(x_{1} x_{2} x_{1}^{-1} x_{2}^{-1}\right)^{-1}\left(x_{3} x_{4} x_{3}^{-1} x_{4}^{-1}\right)^{-1} \\
& =x_{1} x_{2} x_{1}^{-1} x_{2}^{-1} x_{3} x_{4} x_{3}^{-1} x_{4}^{-1} x_{2} x_{1} x_{2}^{-1} x_{1}^{-1} x_{4} x_{3} x_{4}^{-1} x_{3}^{-1} .
\end{aligned}
$$

This sequence has 16 symbols compared to the 22 from $S(4)$ above.

While this savings may not seem significant, the savings becomes substantially more impressive as $n$ grows. If $n$ is a power of two, then $E(1: n)$ has length $n^{2}$, because it consists of two copies of $E(1: n / 2)$ and two copies of $E(n / 2+1: n)$ and because $4(n / 2)^{2}=n^{2}$. Furthermore, in this case, symbols $x_{i}$ and $x_{i}^{-1}$ appear exactly $n$ times in $E(1: n)$ because by induction they appear exactly $n / 2$ times in exactly one of $E(1: n / 2)$ and $E(n / 2+1: n)$. 
If $n$ is not a power of two, we at least have that $E(1: n)$ has length at most $(2 n)^{2}=4 n^{2}$, because $E(1: n)$ only increases if we round up to the next power of two. The integer sequence formed by the length of $E(1: n)$ with $n=1,2,3, \ldots$ is in fact in Neil Sloane's Encyclopedia 8. Ellul, Krawetz, Shallit, and Wang [3. proved that, if $n$ is $b$ larger than the previous power of two, $2^{a}$, then the length of $E(1: n)$ is precisely $\left(2^{a}\right)^{2}+b\left(2^{a+2}-2^{a}\right)$. This formula is always at most $2 n^{2}$. Furthermore, symbols $x_{i}$ and $x_{i}^{-1}$ appear at most $2 n$ times in $E(1: n)$ because each recursion doubles the number of appearances, and there are precisely $\left\lceil\log _{2} n\right\rceil \leq \log _{2} n+1$ recursions, so the number of appearances is at most $2^{\log _{2} n+1}=2 n$. This completes the proof of Theorem 1 .

\subsection{Disjoint Subsets of Nails}

One way to state the most general form of a picture-hanging puzzle is the following: given arbitrary subsets $S_{1}, S_{2}, \ldots, S_{k}$ of $\{1,2, \ldots, n\}$, hang a picture on $n$ nails such that removing all the nails in $S_{i}$ fells the picture, for any $i$ between 1 and $k$, but removing a set of nails that does not include an entire $S_{i}$ leaves the picture hanging. For example, the 1-out-of- $n$ puzzle is the special case of $S_{i}=\{i\}$ for $i=1,2, \ldots, n$. All of the puzzles posed in Section 2 can be represented as particular instances of this general puzzle.

As a warmup to this general form of the puzzle, we first observe that the theory we have developed so far easily solves the special case in which the subsets $S_{1}, S_{2}, \ldots, S_{k}$ are pairwise disjoint. This corresponds to the pegs being divided into different color classes, or "supernails", and the picture falling precisely when an entire color class has been removed. Many puzzles in Section 2 are like this.

Theorem 2. For any partition of $\{1,2, \ldots, n\}$ into disjoint subsets $S_{1}, S_{2}, \ldots, S_{k}$, there is a picture hanging on $n$ nails of length at most $2 k n$ that falls when removing all nails in $S_{i}$, for any $i$ between 1 and $k$, but does not fall when keeping at least one nail from each $S_{i}$.

\section{General Theory}

This section develops a general theory for solving the most general form of the picture-hanging puzzle. Section 3.4 above described one statement of this general form, using subsets, but this turns out to be an inefficient way to represent even relatively simple problems. For example, the $k$-out-of- $n$ puzzle has $\left(\begin{array}{l}n \\ k\end{array}\right)$ subsets of nails that fell the picture, which is exponential for $k$ between $\varepsilon n$ and $(1-\varepsilon) n$. We therefore turn to a more general representation, called "monotone Boolean functions". Although our general solution remains exponential in the worst case, we show in Section 4.4 how this representation allows us to achieve a polynomial solution for $k$-out-of- $n$ in particular.

\subsection{Connection to Monotone Boolean Functions}

For a given picture hanging $p$ on $n$ nails, define the fall function $f_{p}\left(r_{1}, r_{2}, \ldots, r_{n}\right)$, where each $r_{i}$ is a Boolean value (true/1 or false/0), to be a Boolean value 
specifying whether the hanging $p$ falls after removing all $x_{i}$ 's corresponding to true $r_{i}$ 's. For example, a solution $p$ to the 1-out-of- $n$ puzzle has the fall function "is any $r_{i}$ set to true?", because setting any $r_{i}$ to true (i.e., removing any $x_{i}$ ) causes the construction $p$ to fall. In logic, we would write $f_{p}\left(r_{1}, r_{2}, \ldots, r_{n}\right)=$ $r_{1} \vee r_{2} \vee \cdots \vee r_{n}$, where $\vee$ represents OR (logical disjunction).

The most general form of picture-hanging puzzle on $n$ nails is the following: given a desired fall function $f\left(r_{1}, r_{2}, \ldots, r_{n}\right)$, find a picture hanging $p$ with that fall function, i.e., with $f_{p}=f$. Not all such puzzles can be solved, however. Every fall function must satisfy a simple property called monotonicity: if $r_{1} \leq r_{1}^{\prime}$, $r_{2} \leq r_{2}^{\prime}, \ldots$, and $r_{n} \leq r_{n}^{\prime}$, then $f\left(r_{1}, r_{2}, \ldots, r_{n}\right) \leq f\left(r_{1}^{\prime}, r_{2}^{\prime}, \ldots, r_{n}^{\prime}\right)$. Here we view the truth values as 0 (false) and 1 (true), so that false $<$ true. This condition just says that, if the hanging falls when removing certain nails given by the $r_{i}$ 's, and we remove even additional nails as given by the $r_{i}^{\prime}$ 's, then the hanging still falls. A picture hanging cannot "unfall" from removing nails, so monotonicity is a necessary condition on fall functions. For example, it is impossible for a picture hanging to fall from removing any one nail but not from removing more nails.

Monotone Boolean functions are well-studied in combinatorics (through Dedekind's Problem), computational complexity, and computational learning theory, among other fields. It is well-known that they are exactly the functions formed by combining the variables $r_{1}, r_{2}, \ldots, r_{n}$ with the operators AND $(\wedge)$ and OR $(\mathrm{V})$. (In particular, NOT is forbidden.) We can leverage this existing theory about monotone Boolean functions in the context of picture hanging.

\subsection{Arbitrary Monotone Boolean Functions}

In particular, we establish that monotone Boolean functions are exactly the fall functions of picture hangings. We have already argued that every fall function is monotone; the interesting part here is that every monotone Boolean function can be represented as the fall function of a picture hanging. Our construction is exponential in the worst case, but efficient in many interesting cases.

Theorem 3. Every monotone Boolean function $f$ on $n$ variables is the fall function $f_{p}$ of a picture hanging $p$ on $n$ nails. If the function $f$ can be computed by a depth-d circuit of two-input AND and OR gates, then we can construct $p$ to have length $c^{d}$ for a constant $c$. We can compute such $p$ in time linear in the length of $p$. In particular, for functions $f$ representable by a depth- $O(\log n)$ circuit of two-input AND and $\mathrm{OR}$ gates (the complexity class $\mathrm{mNC}^{1}$ ), there is a polynomial-length picture hanging.

Our approach to proving this theorem is to simulate AND and OR gates in a way that allows us to combine them into larger and larger circuits. The most intuitive version of the construction is when the function $f$ is represented as a monotone Boolean formula (as opposed to circuit), which can be parsed into a tree with the $r_{i}$ 's at the leaves and the value of $f$ at the root. As base cases, we can represent the formula $r_{i}$ by the picture hanging $x_{i}$ (or $x_{i}^{-1}$ ), which falls precisely when the $i$ th nail gets removed. We show next that, given picture 
hangings $p$ and $q$ representing two monotone Boolean functions $f$ and $g$, we can construct picture hangings $\operatorname{AND}(p, q)$ and $\mathrm{OR}(p, q)$ representing $f \wedge g$ and $f \vee g$, respectively. While most intuitively applied up a tree representing a formula, the same construction applies to a directed acyclic graph representing a circuit.

Our AND and OR constructions build on two known lemmas from monotone function theory. We start with AND:

Lemma 4. [A. I. Mal'tsev] [4, Lemma 3] For any two words $p, q$ in the free group on $x_{1}, x_{2}, \ldots, x_{n}$, the equation $p^{2} x_{1} p^{2} x_{1}^{-1}=\left(q x_{2} q x_{2}^{-1}\right)^{2}$ is equivalent to the conjunction $(p=1) \wedge(q=1)$.

Using commutator notation, the equation becomes $\left[p, x_{1}\right]=\left[q, x_{2}\right]^{2}$. Because the free group is a group, we can right-multiply the equation by $\left[q, x_{2}\right]^{-2}$ to obtain the equivalent equation $\left[p, x_{1}\right] \cdot\left[q, x_{2}\right]^{-2}=1$.

Lemma 4 states that this equation holds if and only if $p=1$ and $q=1$. Recall that 1 is the fallen state of picture hangings. Thus, the left-hand side

$$
\operatorname{AND}(p, q)=\left[p, x_{1}\right] \cdot\left[q, x_{2}\right]^{-2}=p x_{1} p^{-1} x_{1}^{-1} x_{2} q x_{2}^{-1} q^{-1} x_{2} q x_{2}^{-1} q^{-1}
$$

falls if and only if both $p$ and $q$ fall. This construction is our desired AND.

We now turn to the oR construction:

Lemma 5. [G. A. Gurevich] [4, Lemma 4] For any two words $p, q$ in the free group on $x_{1}, x_{2}, \ldots, x_{n}$, the conjunction of the four equations $\left(p x_{1}^{s} p x_{1}^{-s}\right)\left(q x_{2}^{t} q x_{2}^{-t}\right)=\left(q x_{2}^{t} q x_{2}^{-t}\right)\left(p x_{1}^{s} p x_{1}^{-s}\right)$, for all $s, t= \pm 1$, is equivalent to the disjunction $p=1 \vee q=1$.

Using commutator notation, the equations become $\left[\left[p, x_{1}^{s}\right],\left[q, x_{2}^{t}\right]\right]=1$ for all $s, t= \pm 1$. Lemma 5 states that these equations all hold if and only if $x=1$ or $y=1$. To obtain the conjunction of the four equations, we apply the AND construction above:

$$
\begin{aligned}
& \operatorname{OR}(p, q)=\operatorname{AND}( \operatorname{AND}\left(\left[\left[p, x_{1}\right],\left[q, x_{2}\right]\right],\left[\left[p, x_{1}\right],\left[q, x_{2}^{-1}\right]\right]\right), \\
&\left.\operatorname{AND}\left(\left[\left[p, x_{1}^{-1}\right],\left[q, x_{2}\right]\right],\left[\left[p, x_{1}^{-1}\right],\left[q, x_{2}^{-1}\right]\right]\right)\right) .
\end{aligned}
$$

Thus $\operatorname{OR}(p, q)$ falls if and only if either $p$ or $q$ falls. This construction is our desired OR. The OR formula expands to $144 p$ and $q$ terms, and $474 x_{1}$ and $x_{2}$ terms, for a total of 618 terms.

Analysis. Now we argue that a circuit of depth $d$ results in a picture hanging of length at most $c^{d}$ for a constant $c$. The output of the circuit is the output of some gate, either AND or OR, which has two inputs. Each input can be viewed as the output of a subcircuit of the overall circuit, with smaller depth $d-1$. The two subcircuits may overlap (or even be identical), but we treat them as separate by duplicating any shared gates. By induction on depth, these subcircuits can be converted into picture hangings $p$ and $q$ of length at most $c^{d-1}$. We combine 
these picture hangings via $\operatorname{AND}(p, q)$ or $\mathrm{OR}(p, q)$, according to the output gate type, to obtain our desired picture hanging. The resulting length is at most the maximum length of $p$ and $q$, which is at most $c^{d-1}$, times the number of terms in Equations (1) and (2) defining AND and oR. Thus, setting $c=618$ suffices.

In the base case, the depth-0 circuit has no gates and simply takes the value of a variable $r_{i}$, and we use the picture hanging $x_{i}$, which has length $1=c^{0}$.

This argument gives a $618^{d}$ upper bound on the size of the constructed picture hanging. In fact, only 144 of the 618 terms in (2) are recursive ( $p$ or $q$ ), so the upper bound is $144^{d}$ plus lower-order terms. Thus we obtain Theorem 3

\subsection{Worst-Case Optimality}

Theorem 6. Almost all monotone Boolean functions require length$\Omega\left(2^{n} /(n \log n)\right)$ picture hangings.

This theorem follows from a counting argument, specifically, contrasting the large number of monotone Boolean functions with the relatively small number of picture hangings of a given length.

First we demonstrate a large number of monotone Boolean functions (a standard argument). The vectors $\left(r_{1}, r_{2}, \ldots, r_{n}\right)$ with exactly $n / 21$ 's (and $n / 20$ 's) can all have their function values set independently. There are $\left(\begin{array}{c}n \\ n / 2\end{array}\right)$ such vectors. Thus there are at least $2^{\left(\begin{array}{c}n \\ n / 2\end{array}\right)}$ monotone Boolean functions on $n$ variables.

Next we observe that the number of picture hangings of length $\ell$ is at most $(2 n)^{\ell}$, because there are at most $2 n$ choices for each symbol in the word. (The correct number of choices is $2 n-1$, except for the first, to avoid cancelation.) The number of picture hangings of length at most $\ell$ is $\sum_{i=1}^{\ell}(2 n)^{i}<2(2 n)^{\ell}$.

To represent all monotone Boolean functions, we must have $2(2 n)^{\ell} \geq 2^{\left(\begin{array}{c}n \\ n / 2\end{array}\right)}$. Taking $\log _{2}$ of both sides, we must have $1+\ell\left(1+\log _{2} n\right) \geq\left(\begin{array}{c}n \\ n / 2\end{array}\right)$. Asymptotically, $\left(\begin{array}{c}n \\ n / 2\end{array}\right) \sim 2^{n} \sqrt{\frac{2}{\pi n}}$. Thus we must have $\ell \sim 2^{n} \sqrt{\frac{2}{\pi n \log _{2} n}}$. A standard "almost every" argument completes the proof of Theorem 6 .

\section{$4.4 k$-out-of- $n$}

Theorem 7. For any $n \geq k \geq 1$, there is a picture hanging on $n$ nails, of length $n^{c^{\prime}}$ for a constant $c^{\prime}$, that falls upon the removal of any $k$ of the nails.

We simply argue that the monotone Boolean function "are at least $k$ of the $r_{i}$ 's true?" is in the complexity class $\mathrm{mNC}^{1}$, that is, can be represented by a logarithmic-depth binary circuit. The idea is to sort the $r_{i}$ values, again viewing Boolean values as 0 (false) and 1 (true), using a logarithmic-depth sorting network. The result of this sorting is a sequence of $j 0$ 's followed by a sequence of $n-j 1$ 's. Our goal is to determine whether $n-j \geq k$. To do so, we would simply look at the $(n-k+1)$ st item in the sorted order: if it is 1 , then there at least $k 1$ 's, and otherwise, there are fewer. 


\section{$5 \quad$ Spectating Is Hard}

Imagine we turn the tables and, instead of considering the magician's challenge in hanging a picture on $n$ nails with certain properties, we consider the spectator's challenge of choosing which nails to remove. A natural objective, if the spectator is shy and wants to get off stage as quickly as possible, is to remove as few nails as possible in order to make the picture fall. Unfortunately for the spectator, for a given picture hanging, this problem is NP-complete and hard to approximate:

Theorem 8. For a given picture hanging on $n$ nails, it is NP-complete to decide whether there are $k$ nails whose removal fells the picture, and it is hard to approximate the minimum number of nails within some $\varepsilon \log n$ factor.

We can similarly argue that it is NP-hard for the attention-hoarding spectator who aims to maximize the number of nails to remove before felling the picture hanging. By the same reduction, this problem becomes finding a set of elements that hit every set in the collection $\mathcal{S}$, which is the Hitting Set problem. Reversing the roles of elements and sets, we have the identical Set Cover problem. Inapproximability no longer follows because the objectives are reversed.

\section{Open Problems}

Several interesting open questions remain about optimality of our constructions. Does the 1-out-of- $n$ picture hanging puzzle require a solution of length $\Omega\left(n^{2}\right)$ ? What is the complexity of finding the shortest picture hanging for a given monotone Boolean function? For the spectator, is there an $O(\log n)$-approximation algorithm for removing the fewest nails to fell the picture hanging?

Acknowledgments. We thank Jason Cantarella for helpful early discussions, and Kim Whittlesey for pointing out reference [10. J. Mitchell is partially supported by NSF grant CCF-1018388.

\section{References}

1. H. Brunn. Über Verkettung. In Sitzungsberichte der Bayerische Akad. Wiss., 22:77-99, 1892.

2. E. D. Demaine, M. L. Demaine, and R. Uehara. Any monotone Boolean function can be realized by interlocked polygons. In CCCG 2010, 139-142.

3. K. Ellul, B. Krawetz, J. Shallit, and M. Wang. Regular expressions: new results and open problems. J. Autom. Lang. Comb., 9(2-3):233-256, 2005.

4. G. S. Makanin. Decidability of the universal and positive theories of a free group. Mathematics of the USSR-Izvestiya, 25(1):75-88, 1985.

5. E. Pegg Jr. http://www.mathpuzzle.com/hangingpicture.htm

6. D. Rolfsen. Knots and Links. Publish or Perish, Inc., Houston, Texas, 1976.

7. T. Sillke. http://www.mathematik.uni-bielefeld.de/ $\sim_{\text {sillke/PUZZLES/quantum/B201 }}$

8. N. J. A. Sloane. Sequence A073121. In On-Line Encyclopedia of Integer Sequences. August 2002. http://www.research.att.com/projects/OEIS?Anum=A073121

9. A. Spivak. Brainteasers B 201: Strange painting. Quantum, p. 13, May/June 1997.

10. T. Stanford. Brunnian braids and some of their generalizations. Bull. Lond. Math. Soc.. To appear. arXiv:math.GT/9907072, http://arXiv.org/abs/math/9907072

11. P. G. Tait. On knots. Trans. Royal Society of Edinburgh, 28:145-190, 1876.

12. M. vos Savant. Ask Marilyn. PARADE, 2001. Posed June 10 and solved June 17. 